\title{
VISIBILITY OF GRAPHICAL CHARACTER E-MAIL IN MULTIPLE LANGUAGES ON MOBILE PHONES
}

\author{
Satoshi Hasegawa * $\uparrow$, Yuki Ire **, Masako Omori ${ }^{* *}$, Shohei Matsunuma ***, Masaru Miyao ** \\ * Nagoya Bunri University, ** Nagoya University, *** Telecom Express Co., Ltd \\ $\dagger$ e-mail : hasegawa@nagoya-bunri.ac.jp
}

\begin{abstract}
Although multilingual information is required by many foreigners in Japan, ordinary mobile phone (MP) models in this country do not support multilingual characters such as Korean or Chinese. Graphical e-mail systems in MPs for sending digital photographs are also useful in sending character mail with unsupported fonts. This paper presents research on the visibility of Korean, Chinese, and Japanese graphical characters on the liquid crystal displays (LCDs) in MPs, by measuring the variables of reading time and visual distance. The dependent relationship between size and visibility of the characters as well as the importance of the quality of the LCD are described. Visibility is also affected by the age of the people using MPs.
\end{abstract}

\section{INTRODUCTION}

\section{Graphical Character E-Mail in Mobile Phones in Japan}

Mobile phones (MPs) with digital cameras have spread widely in Japan. Image e-mail systems are usually used for sending natural images such as scenery or portrait photographs. Image e-mail is also useful in sending characters although this is not its original usage. By using graphical images, characters with unsupported fonts can be sent. An image data format supported in the e-mail system of many types of MPs was developed by Joint Photographic Experts Group (JPEG) for photographic images. The JPEG format is not necessarily suitable to representing characters. The visibility of graphical characters in MPs should be studied, including the influence of the quality of the liquid crystal display (LCD) in the MPs.

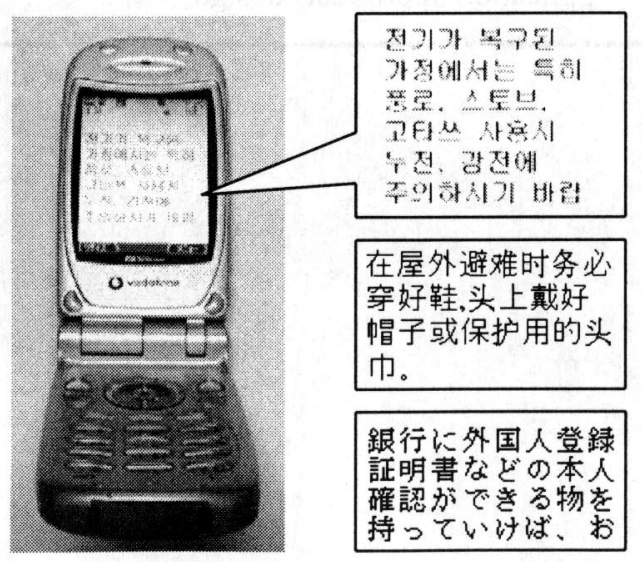

Figure 1. Multilingual graphical character e-mail

\section{Multilingual Information}

Multilingual information will be helpful to many foreign residents in Japan. Emergency information in particular should be accessible to people of all nationalities (Sato, 2004). However, ordinary models of MPs in Japan support only Japanese and alphanumeric characters. Graphical character e-mail enables MPs in Japan to send multilingual characters such as Korean and Chinese, as shown in Figure 1.

\section{Users' Age and the Size of Characters}

Disaster prevention information or other important messages are necessary for persons of all age groups, as well as all nationalities. A previous report (Omori, 2002) showed that small fonts in MPs were hard for elderly people to read. The dependence of visibility on character size and users' age should be further researched for graphical characters.

\section{METHOD}

\section{Subjects}

The four examinations described below were conducted. Each subject was a native speaker of one of the following

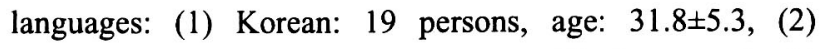
Chinese: 10 persons, age: 27.6 \pm 4.1 , (3) Japanese-1: 24 persons, age: $23.5 \pm 2.2$, (4) Japanese-2: 88 persons, age: $46.3 \pm 17.9$, including 33 elderly $60-79$ years of age. 


\section{Specifications of MPs and Their LCDs}

The following MPs were used in the examinations. Type A: J-SH53, TFT (240), Type B: J-SH07, TFT (120), Type C: J-SA51, TFT (132), Type D: J-SA03, STN (120), (LCD width [dot]). Type $A$ is higher in luminosity and resolution than $B$. Type $C$ has higher specifications than $D$.

\section{Graphical Characters}

Korean, Chinese, and Japanese characters were presented as JPEG format image data. Several data sets, including different contents, were made for each language and each resolution (120 dot width or 240 dot width). Each data set was prepared in three different character sizes (similar to typical font size, respectively) but the same number of characters (see Figure 2). Each data file was at less than $6 \mathrm{~KB}$ (for MP type B, C, D) or $30 \mathrm{~KB}$ (type A) in size, in order to send as an MP e-mail.

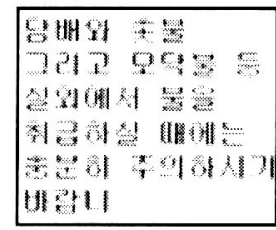

(a) size $\mathrm{L}$

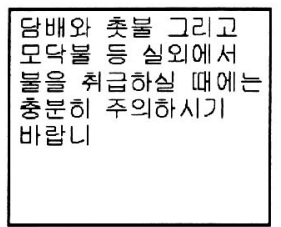

(b) size $M$

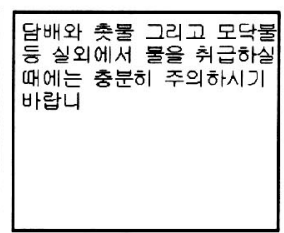

(c) size $\mathrm{S}$
Figure 2. Examples of Graphical Characters

\section{Experiment and Measurements}

Samples were displayed in rotated order so that no subject read the same contents. Reading time and visual distance between eyes and LCD were measured. After each reading, subjects evaluated visibility from 1 (very hard to read) to 5 (very easy to read).

\section{RESULTS}

Results for the first three groups are shown in Figure 3 (Korean), Figure 4 (Chinese), and Figure 5 (Japanese). Figure 6 shows the dependency of visibility on subject age (4). The results of the elderly subjects (age: 60-79) from group (4) are shown in Figure 7. Significant differences by the size of characters are seen in subjective evaluation of all of the languages, especially in the lower quality type of MP. Reading speed and visual distance decrease as the character size becomes smaller (Figure 5). Visibility is correlated with age (Figure 6). The worst reading speed is seen with size $S$ among the elderly subjects (Figure 7).

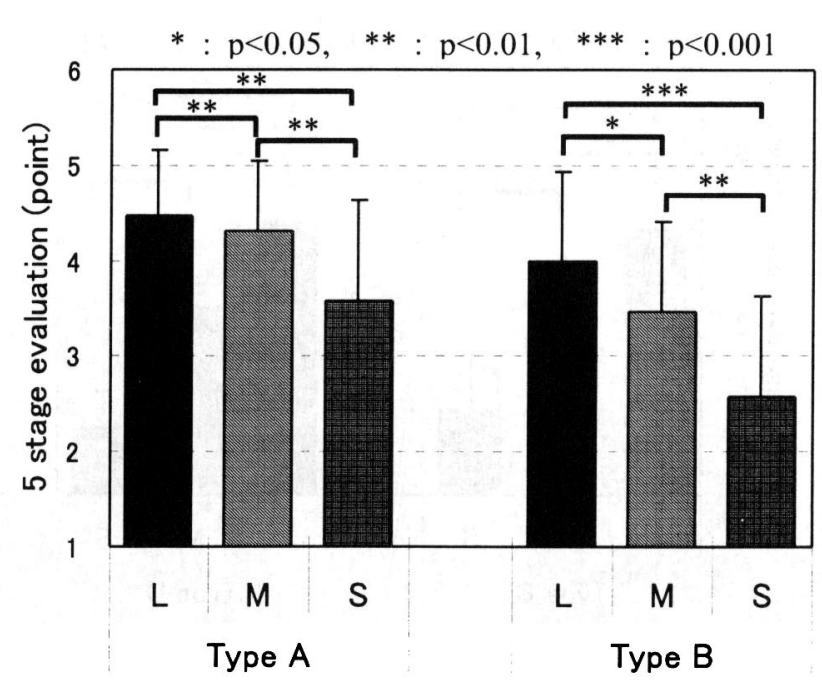

(a) Subjective Evaluation

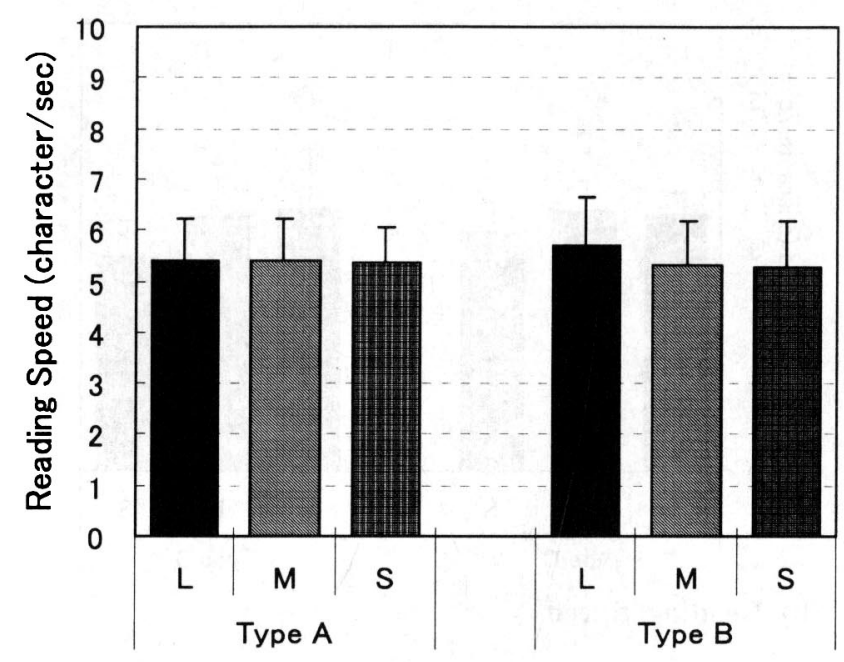

(b) Reading Speed

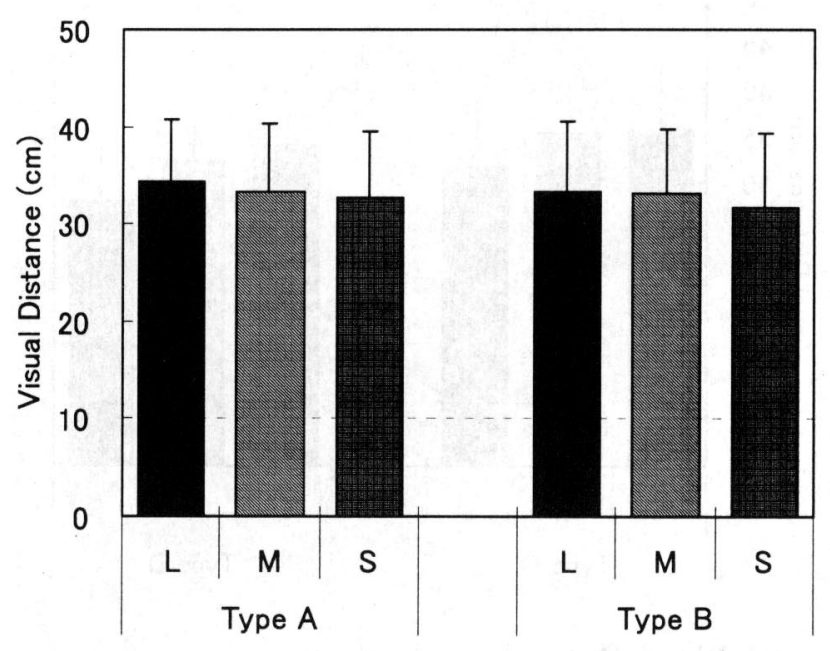

(c) Visual Distance

Figure 3. Visibility of Korean Characters 


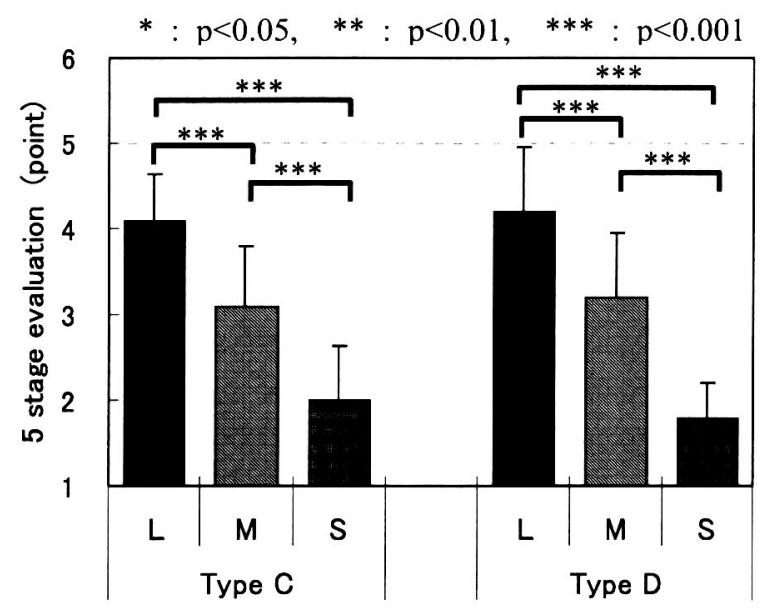

(a) Subjective Evaluation

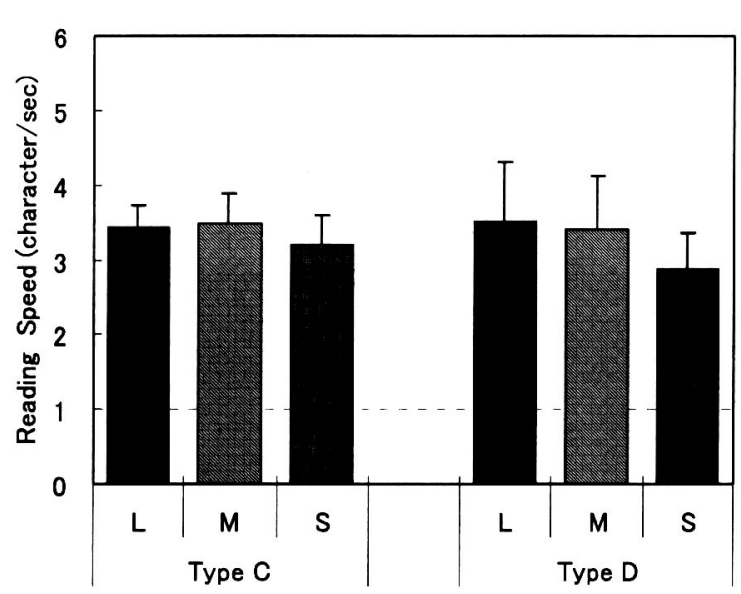

(b) Reading Speed

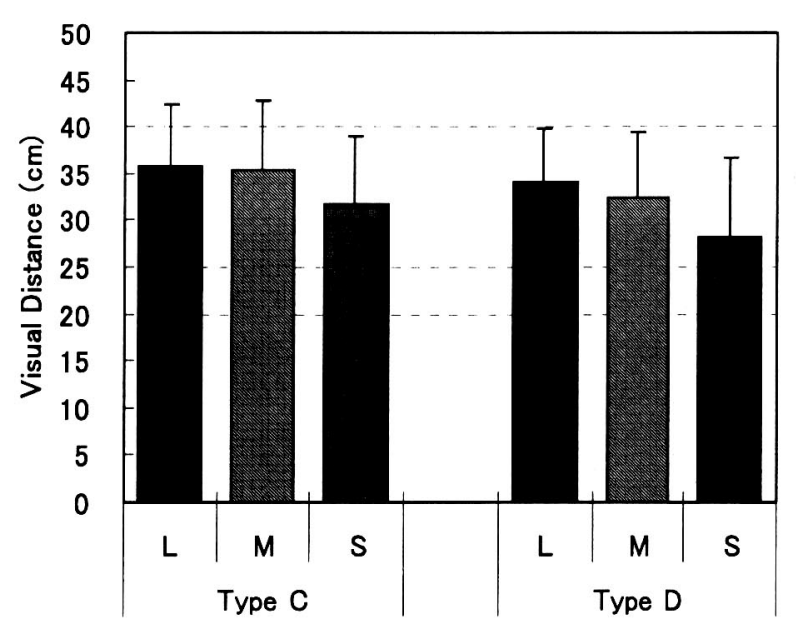

(c) Visual Distance

Figure 4. Visibility of Chinese Characters

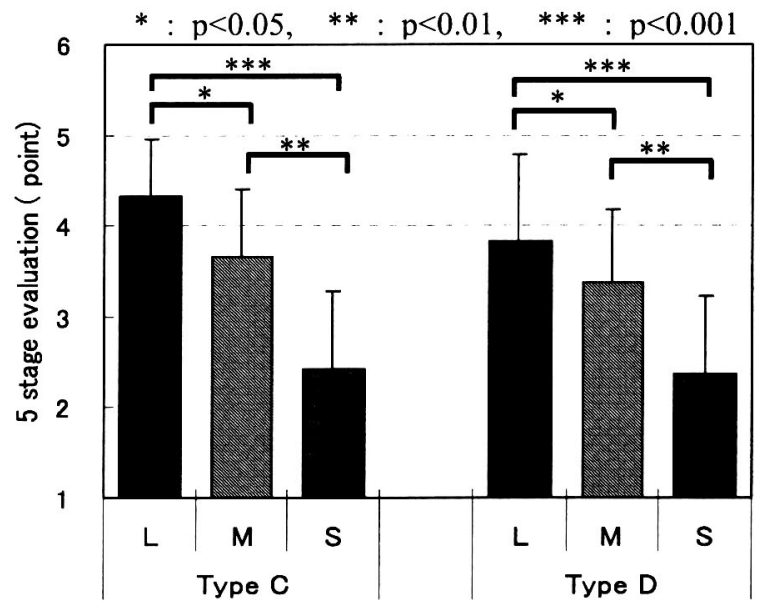

(a) Subjective Evaluation

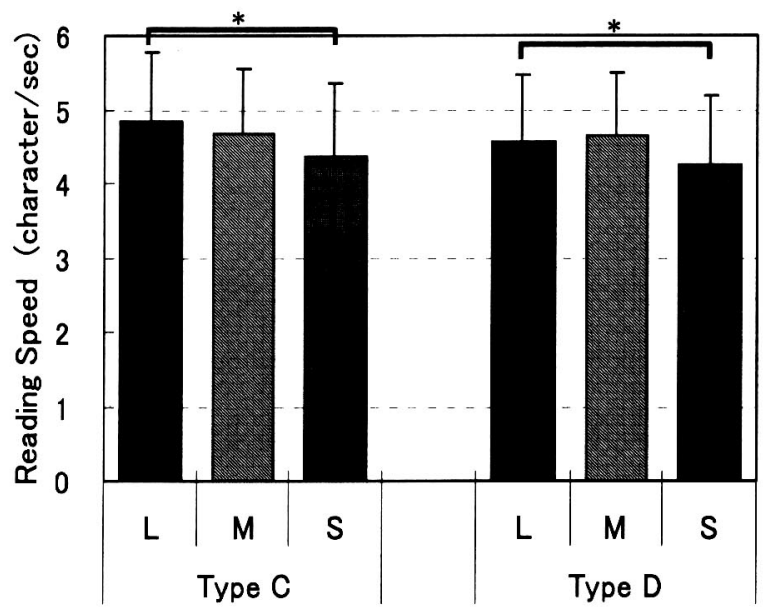

(b) Reading Speed

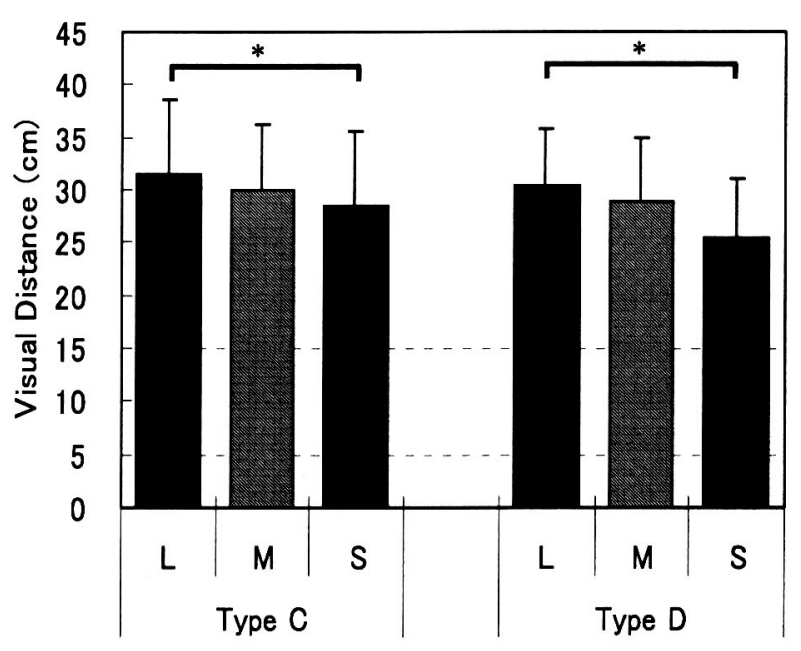

(c) Visual Distance

Figure 5. Visibility of Japanese Characters 

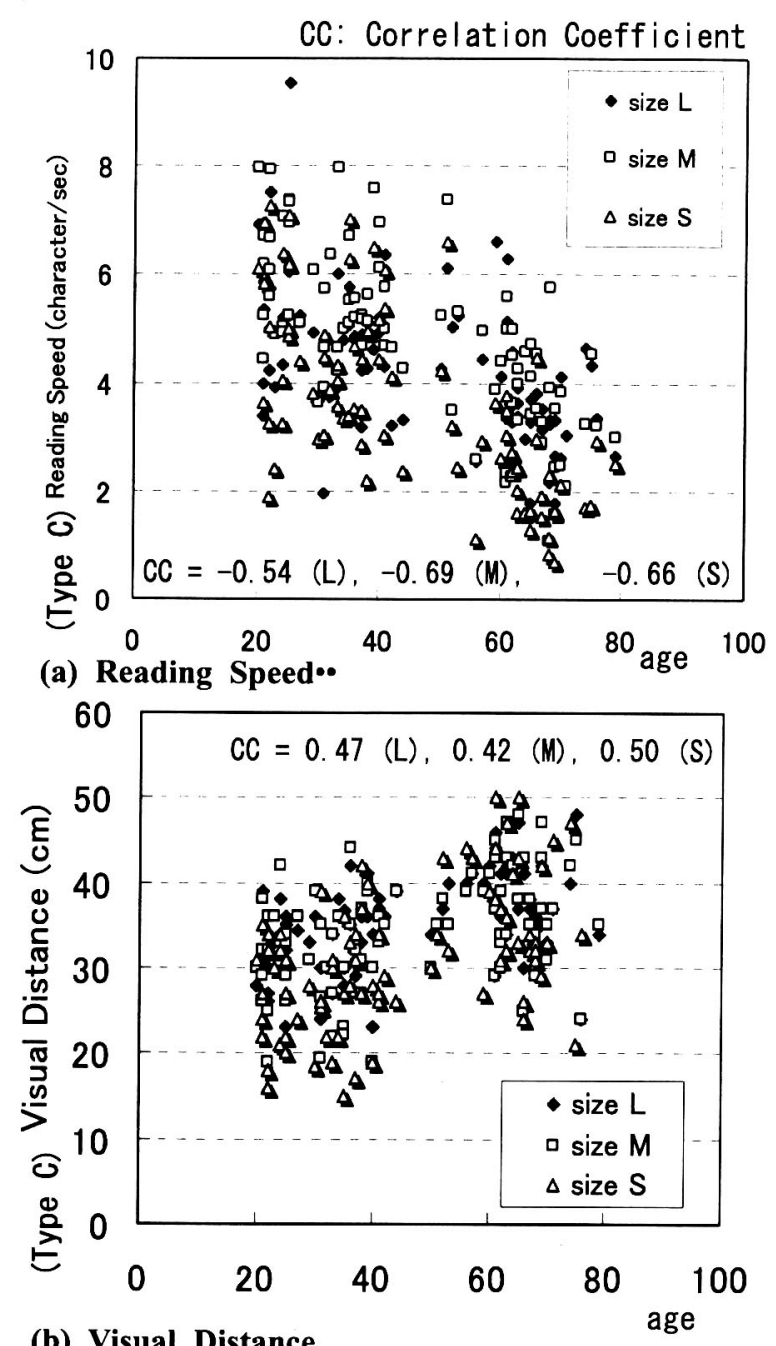

(b) Visual Distance

Figure 6. Dependence of Visibility on Age

\section{CONCLUSION}

The possibility of graphical character e-mail in MPs was shown for the Korean, Chinese, and Japanese languages. However, it must be noted that visibility deteriorates as the character size become smaller, especially when the user is an elderly person.

\section{REFERENCES}

Sato, K. et al. 2004. Multilingual and ubiquitous information system for disasters. ESK and JES Joint Symposium 2004, 5-Jun-04 (in printing)

Omori, M. et al. 2002. Visibility and characteristics of the mobile phones for elderly people, Behavior \& Information Technology, 21(5), 313-316

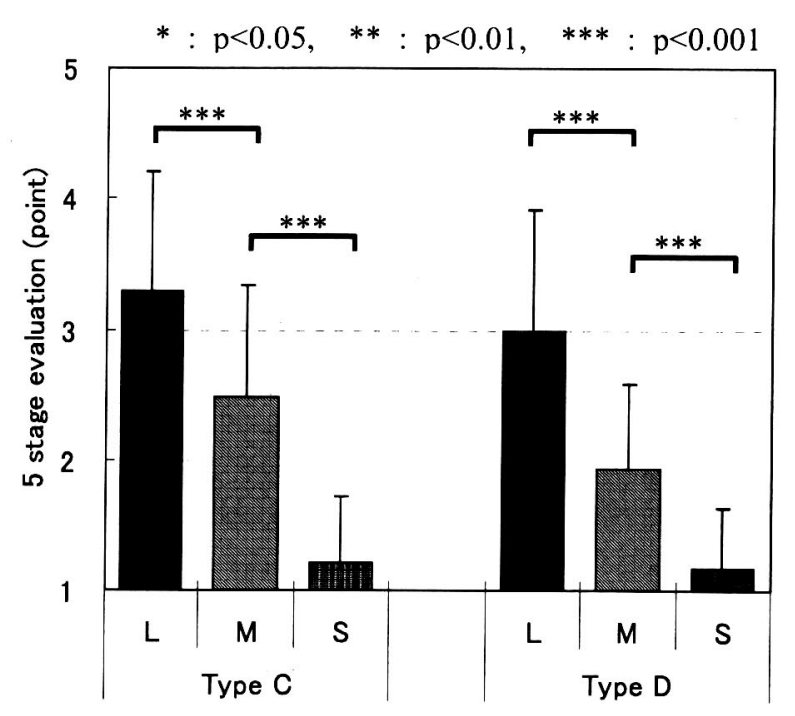

(a) Subjective Evaluation.•

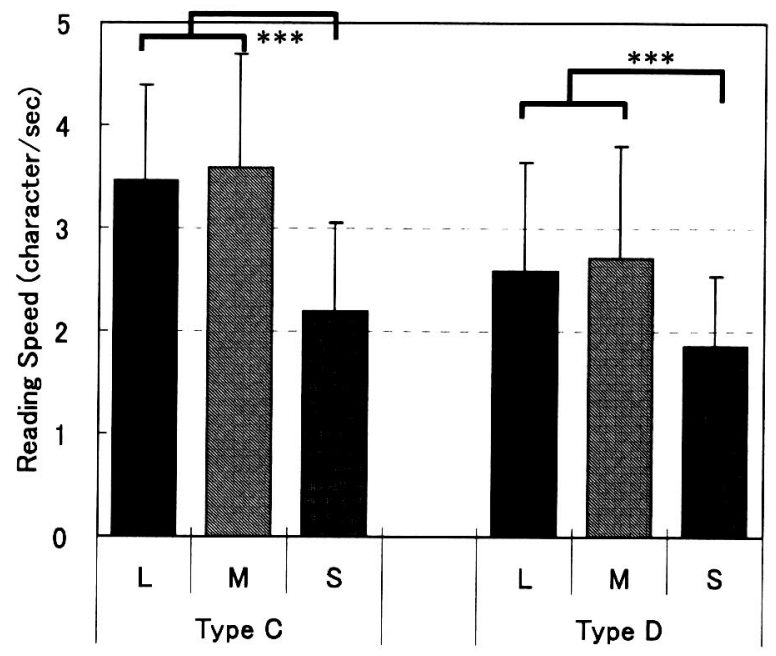

(b) Reading Speed.•

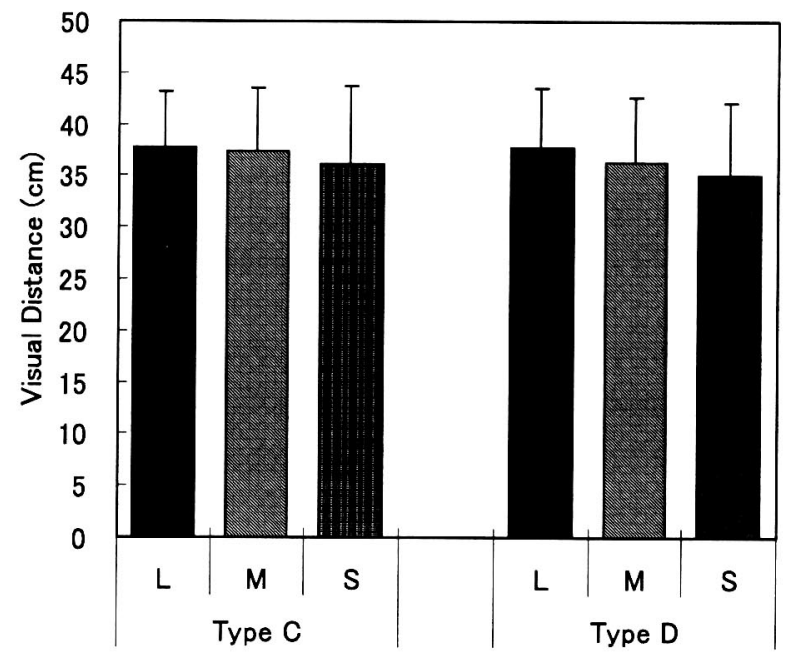

(c) Visual Distance

Figure 7. Visibility of Japanese Characters by Elderly 